\title{
In the news
}

VITAMIN C:

FRIEND OR FOE?

Controversy has dogged the use of vitamin $\mathrm{C}$ supplements during cancer therapy, and research from the Memorial Sloan-Kettering Cancer Center has brought this once more into the public eye.

A preclinical study has shown that pretreatment of tumour cells with dehydroascorbic acid has a protective effect against a variety of anticancer agents - including imatinib, doxorubicin, cisplatin, vincristine and methotrexate - both in vitro and in mice. The work published in Cancer Research suggested that vitamin C inhibited drug-induced mitochondrial membrane depolarization. Speaking of his study, Dr Mark Heaney explained, "Vitamin C appears to protect the mitochondria from extensive damage, thus saving the cell" (http://nytimes. com/ 1 Oct 2008). The build-up of the vitamin $C$ within tumour cells could also be seen in those taking it in large supplemental doses, but the current work is yet to be extended to patients.

Dr Heaney emphasized that vitamin $C$ is necessary in the diet but stated, “I don't recommend taking supplemental vitamin $\mathrm{C}$ during that period of time that my patients are receiving chemotherapy" (http://www. reuters.com/ 1 Oct 2008). The amounts of vitamin C used in the study were extremely high, but equivalent doses can be bought in health stores. Pamela Mason from the Health Supplements Information Service advised caution, recommending that anyone undergoing cancer treatment "seek the advice of their doctor or pharmacist before taking any product not prescribed by their doctor" (http:// www.telegraph.co.uk/ 1 Oct 2008).

However, Dr Chi Van Dang from Johns Hopkins University emphasized that the possible beneficial effects of the molecule should not be forgotten: "Additional studies are necessary regarding whether vitamin $\mathrm{C}$ as a single agent could prevent cancer or the recurrence of cancer once treated" (http://www.washingtonpost. com/ 1 Oct 2008). 